\title{
Molecular Dynamics Simulations of Coulomb Explosion
}

\author{
E. M. Bringa
}

This article was submitted to

Swift Heavy lons in Matter, Taormina, Italy, May 21-25, 2002

\section{May 17, 2002}

U.S. Department of Energy

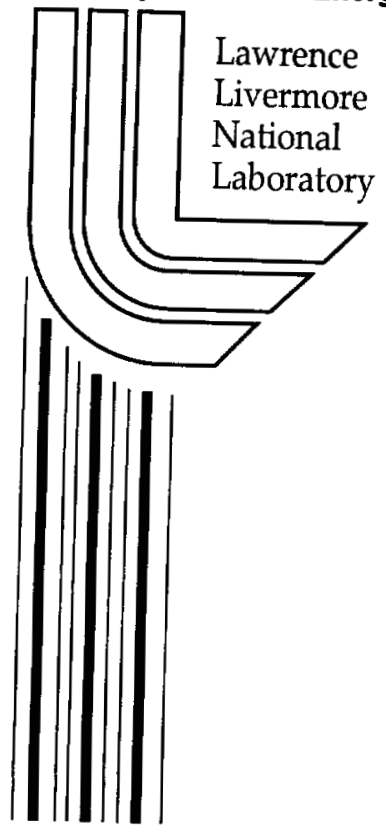





\section{DISCLAIMER}

This document was prepared as an account of work sponsored by an agency of the United States Government. Neither the United States Government nor the University of California nor any of their employees, makes any warranty, express or implied, or assumes any legal liability or responsibility for the accuracy, completeness, or usefulness of any information, apparatus, product, or process disclosed, or represents that its use would not infringe privately owned rights. Reference herein to any specific commercial product, process, or service by trade name, trademark, manufacturer, or otherwise, does not necessarily constitute or imply its endorsement, recommendation, or favoring by the United States Government or the University of California. The views and opinions of authors expressed herein do not necessarily state or reflect those of the United States Government or the University of California, and shall not be used for advertising or product endorsement purposes.

This is a preprint of a paper intended for publication in a journal or proceedings. Since changes may be made before publication, this preprint is made available with the understanding that it will not be cited or reproduced without the permission of the author.

This work was performed under the auspices of the United States Department of Energy by the University of California, Lawrence Livermore National Laboratory under contract No. W-7405-Eng-48.

This report has been reproduced directly from the best available copy.

Available electronically at http://www.doc.gov/bridge

Available for a processing fee to U.S. Department of Energy

And its contractors in paper from

U.S. Department of Energy

Office of Scientific and Technical Information

P.O. Box 62

Oak Ridge, TN 37831-0062

Telephone: (865) 576-8401

Facsimile: (865) 576-5728

E-mail: reports@adonis.osti.gov

Available for the sale to the public from

U.S. Department of Commerce

National Technical Information Service

5285 Port Royal Road

Springfield, VA 22161

Telephone: (800) 553-6847

Facsimile: (703) 605-6900

E-mail: orders@ntis.fedworld.gov

Online ordering: http:L/www.ntis.gov/ordering.htm

OR

Lawrence Livermore National Laboratory

Technical Information Department's Digital Library

http://www.llnl.gov/tid/Library.html 



\title{
Molecular Dynamics Simulations of Coulomb Explosion
}

\author{
E. M. Bringa ${ }^{1}$ \\ Lawrence Livermore National Laboratory \\ Chemistry and Material Sciences Directorate, MSTD \\ L353, PO. Box 808, Livermore, CA 94550
}

\begin{abstract}
A swift ion creates a track of electronic excitations in the target material. A net repulsion inside the track can cause a "Coulomb Explosion", which can lead to damage and sputtering of the material. Here we report results from molecular-dynamics (MD) simulations of Coulomb explosion for a cylindrical track as a function of charge density and neutralization/quenching time, $\tau$. Screening by the free electrons is accounted for using a screened Coulomb potential for the interaction among charges. The yield exhibits a prompt component from the track core and a component, which dominates at higher excitation density, from the heated region produced. For the cases studied, the number of atoms ejected per incident ion, i.e. the sputtering yield $Y$, is quadratic with charge density along the track as suggested by simple models. $Y(\tau=0.2$ Debye periods $)$ is nearly $20 \%$ of the yield when there is no neutralization $(\tau \rightarrow \infty)$. The connections between 'Coulomb explosions', thermal spikes and measurements of electronic sputtering are discussed.
\end{abstract}

Key words: Coulomb explosion, sputtering, tracks, molecular dynamics PACS: 79.20.A, 81.65.C, 34.50.Fa, 79.20.R 


\section{Introduction}

When a solid is bombarded by a fast ion, a track of excitations is formed [1]. The incident ion promotes atoms to excited states, and also creates electronhole pairs. Some electrons are expelled from the track as fast delta electrons, creating other electron-hole pairs in a time scale of $10^{-17}-10^{-16} \mathrm{~s}$. They gradwally thermalize, screening the holes, and finally recombine with the holes in times which vary from $\sim 10^{-16}$ to $10^{-6} \mathrm{~s}$ depending on the material. If hole mobility is low and recombination slow, the holes can repel each other leading to a "Coulomb explosion" (CE), in which the charges acquire large velocities and transfer energy to neighbors. CE has been suggested to produce track amorphization, material damage [1-3], surface modification [4], and particle ejection (sputtering) [5-7]. However, it is not clear whether or not the Coulomb repulsion in the track core lasts long enough to produce significant effects and whether or not it can quantitatively described the energy deposition in the track core.

Although CE has been thought to play a role in many experiments on swift ion bombardment, there are few direct simulations of this process. In a recent paper [8], we presented $\mathrm{MD}$ simulations of $\mathrm{CE}$ focusing on the comparison of CE and "thermal spike" (TS) models. Here we explain our simulations in more detail and present some new results. We first review some existing models of Coulomb explosion, then give the details of our model computer simulation, which includes screening and neutralization/quenching effects, and finally present our results.

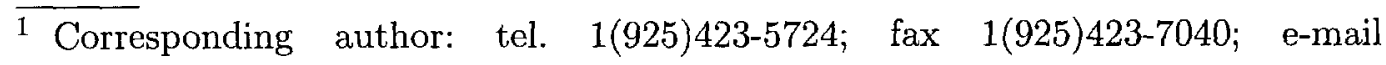
ebringa@llnl.gov 


\subsection{Models of Coulomb Explosion}

In 1965 Fleischer, Price and Walker (FPW) developed their now well-known "ion explosion model" to account for track registration in solids [1]. In this model the large positive charge density in the track core leads to a transformation of the electrostatic potential energy into kinetic energy of the charged atoms. These in turn transfer kinetic energy to neighboring atoms. This model appeared to successfully predict when ion tracks could be "registered", but was not used to describe surface effects. Damage in the track was related to the ionization per unit path length, $d J / d x$, rather than to the energy loss per unit path length, $d E / d x$. FPW showed that analytic TS models [9] could not explain track registration data. These TS models were overly simplified [10-12] and the experimental data were not comprehensive. Models and experiments [3] appear to suggest that even in metals, where screening by the conduction electrons is rapid, CE can deposit enough energy in the lattice to produce a shock wave which produces defects.

Haff [6] first used the ion explosion concept to estimate a sputtering yield, $Y$, suggesting that $Y \propto(d J / d x)^{2}$. Subsequently, Tombrello and coworkers developed a "thermalized ion explosion model" [13]. In this model the CE leads to a localized high temperature region, and sputtering was then calculated using an analytic spike model. This gave $Y \propto(d J / d x)^{4}$ when the radius of the spike, $r_{c y l}$, was such that $r_{c y l} \propto d J / d x$. This model appeared to fit certain data over a limited range of $d J / d x$ [13]. Ritchie and Claussen [14] suggested that the secondary electrons from the ion track heated the neutrals by collisions. Later, Ritchie et al. [15] developed a numerical model to track all charged particles, including electrons, assuming complete ionization within a certain 
time-varying radius. A class of models which are a variation on the CE model $[16,17]$ assume that the escape of the electrons from the track changes the effective potential among atoms. This leads to an instability which increases the kinetic energy of the atoms in the track. Ejection may then be direct or due to the resulting thermal spike. Such a model has been used to explain recent gigantic sputtering data for GaAs [7]. A CE model of sputtering, applied to ionic crystals, was also presented by Bitenskii et al. [18], where the value of the yield depended on a fitting parameter and on the value of the neutralization time for the charges.

\subsection{Coulomb Explosion Simulations}

We use MD simulations to follow the evolution of the "ionized track" in a Lennard-Jones (LJ) fcc solid. Details of the MD code have been described elsewhere $[10,8]$. Because the cohesive energy and spatial dimensions scale with the LJ parameters, results for other solids can be obtained using the appropriate number density $n$ and cohesive energy $U$. The Debye period, $\tau_{D}$, gives a characteristic time scale for phonons and also scales with $n$ and $U$. MD simulations of CE have been performed for small clusters [19], and there have also been other simulations where the initial velocity of the atoms induced by Coulomb repulsion was assumed to be known and the motion of atoms was then followed $[20,21]$. CE in $\mathrm{LiF}$ due to two neighbor holes has been reported [22], but the first MD simulations of CE in the solid phase with more than 2 charges [23] were carried out only for small 2D samples. Cheng and co-workers [24] have reported large-scale 3-D simulations for $\mathrm{CE}$ in $\mathrm{Si}$, with a charge

configuration that extended a few layers below the surface, such as produced by a slow, highly charged projectile. In this work we deal with different initial 
conditions. For a swift ion the track length is much larger than the other relevant sizes. Not all atoms inside the track are ionized by the passage of the ion, and the number of ionizations changes with the stopping power of the incoming ion. Finally, the charges in the track can have a lifetime that can be much shorter than the simulation time, so neutralization is included.

In order to simulate the CE process within an ion track, a specified number of atoms within a cylinder of radius $r_{t r}$ were given a net charge at time $t_{o}$. Atoms were chosen randomly for each simulation, giving a mean number of charges per layer $N_{c h}$, which is proportional to $d J / d x, l_{s} d J / d x=N_{c h}$, where $l_{s}$ is the layer spacing. Most simulations are performed for $r_{t r} \sim l_{s}$, which gives a maximum of 2 charges per layer. Runs for $r_{t r} \sim 2.5 l_{s}$ show the same behavior at early times. Here we simulate an 'ionization track' in which the holes have low mobility and are not fully screened during the time atomic displacements occur. Even if the holes are neutralized, a track of excited atoms will have overlapping charge clouds that act repulsively $[5,16,17]$. Therefore, we do not distinguish between closely spaced, partially screened holes or interacting excited atoms. We describe the repulsion between such excited ('ionized') atoms using a screened Coulomb potential in addition to the LJ potential. The screening function was chosen to be an exponential, giving the Yukawa form for the potential $V=\left(e^{2} / r\right) \exp (-r / a)$, where $a$ is an average screening constant. This interaction has a large cut-off at $r_{c u t-C o u l}=7 a$ in order to provide energy conservation. Coulomb interactions in polar insulators, such as water, are typically neglected due to the high dielectric constant. In non-polar materials, screening is small when there are no excited ('free') electrons. For our simulations we assume that the produced 'free' electrons screen the ions in the track, creating a very hot plasma [14]. $a$ can be taken to be the Debye 
screening length [25], which depends on the local electron density, $n_{e}$, and electron thermal velocity, $v_{T}, a=\lambda_{D}=v_{T} / \omega_{p}=\sqrt{\epsilon k_{B} T / n_{e} e^{2}}$, where $\epsilon$ is the dielectric constant. A Yukawa potential with Debye screening has been used to calculate the screening among atoms of $\mathrm{C}_{60}$ clusters which 'Coulomb-explodes' on penetrating a solid [26]. The classical Debye length should be replaced by the Thomas-Fermi length, $a_{T F}$, for certain densities/temperatures of the electrons [25] but, for the cases considered here, $\lambda_{D}$ is more appropriate. Using $n_{e}=0.01 / \AA^{3}$ and $k_{B} T=10 \mathrm{eV}$ gives $\lambda_{D} \sim l_{s} \sim 3 a_{T F}$. The screening in fact varies with time, due to the cooling of the electrons to the lattice, and depends on the local environment. Recombination models (for instance Auger [27], columnar [28], or geminate recombination [29]), that predict the changes of the electronic density could be included in future studies. If the screening length does not change significantly during the time scale at which CE occurs, then MD simulations with a constant value of a can be carried out. Monte Carlo simulations of a track of holes and electrons in amorphous solid Ar by R. Vidal and J. Ferrón [30] show that the electron density is roughly a Gaussian by $t \sim 1 \mathrm{fs}$, and that it does not change significantly during several fs, when the simulation stops. In molecular solids, the electrons will suffer significant inelastic collisions, leading to an even narrower spatial distribution around the initial track. Neutralization may also change the distribution of electrons before $\mathrm{CE}$ is effective. In order to test the validity of using a constant screening length, $a$, track simulations including inelastic energy loss have been performed and it was found that the density did not change significantly for times of the order of $\tau_{D}$ and energy losses appropriate for ice [8,31].

Neutralization in the track core during sputtering should be taken into account. To evaluate its effect on the sputtering yield, we allow the number of 
charges within the track to decrease exponentially with a quenching/neutralization time $\tau$, as $\exp \left[-\left(t-t_{o}\right) / \tau\right]$. Screening and neutralization are closely related. That is, a large electron density which heavily screens the charges is similar to electrons which neutralize the charges. At large electron densities hole neutralization is initially fast, but the resulting reduction in the screening rapidly slows the process $[8,31]$. Estimates of neutralization times vary by orders of magnitude. Ion-induced electron emission spectra can give insight into the magnitude of the neutralization times $[33,34]$.

The interaction of the charged atoms with the neutral atoms was left unchanged in our simulations. The neutrals, of course, contribute to the screening, but in test simulations the effect of the polarization of the neutrals on lattice heating was small. Particles are sputtered if they cross a plane $2 r_{\text {cut }}^{\text {coul }}$ above the surface. The number of atoms ejected per run is called the yield. Only results for the (001) surface are shown. The samples had an open top surface to allow for sputtering. Sample sizes $\left(3 \times 10^{4-5}\right.$ atoms $)$ and simulation times $(15-80 \mathrm{ps})$ were adjusted to the size of $a$ and $d J / d x$. Extending times by tens of picoseconds, doubling the thickness, or changing the boundary conditions did not change these results. Sample depth was chosen to be at least twice the cut-off radius for the Yukawa potential. Yields from individual simulations were averaged over $\sim 10-100$ 'ionization' distributions because the spread in the size of the yield is broad, especially for small $(d J / d x)$ where sputtering only occurs when two excitations are produced close together at the surface $[5,6]$. 


\section{Results}

We find that the sputtering yield, $Y$, for a given screening constant, $a$, and for a given neutralization time, $\tau$, is roughly given by:

$$
\begin{aligned}
& Y(\tau=\infty) \quad \approx 14.1 \ln \left[1.3 a / r_{t r}\right]\left[l_{s}(d J / d x)\right]^{2} \\
& Y\left(\tau \lesssim 50 T_{D}\right) \approx Y(\tau=\infty) \exp \left[-0.75\left(-\tau_{D} / \tau\right)^{0.50}\right]
\end{aligned}
$$

$Y(\tau=\infty)$ indicates the yield obtained with static screening but no neutralization. The quadratic dependence with $(d J / d x)$ predicted by several models is only valid if the screening length $a$ is assumed to be constant for different values of $(d J / d x)$. Fig. 1 shows the sputtering yield as a function of $a$, for $a$ in the range $l_{s}$ to $5 l_{s}$. Values of the screening constant $a$ smaller than the nearest neighbor distance give very small yields since the repulsion between excited species is negligible. The logarithmic dependence with $a$ in Eq. 1, seen in Fig. 1, agrees with analytic predictions [5]. Fig. 2 shows the yield from MD simulations as a function of $\tau$ together with the fit from Eq. 1 for a number of cases. For $\tau=0.2 \tau_{D}$ the yield is nearly $20 \%$ of the yield for $Y(\tau=\infty)$, indicating that $\mathrm{CE}$ could be important in ejection processes if neutralization (quenching) time $\tau \gtrsim 2 \tau_{D}$.

Fig. 3 shows a series of snapshots of a CE simulation and illustrates ejection due to "direct" Coulomb repulsion: two large red circles (hot charged particles) being ejected by 0.26 ps. Later ejection involves neutrals (small circles) which are both hot and "cold". In the last snapshot the crystal structure near the surface shows some damage, but it, is well reconstructed away from the surface. 
Fig. 4(a) and (b) show depth and radial distributions of ejecta, normalized to unit area, for two different neutralization times. The longer the neutralization time, the deeper and farther from the track the ejecta can originate. Fig 5 (a) and (b) show the kinetic energy (KE) distribution of ejecta for the same neutralization times as in Fig. 4. At large KE, $E \geq 10 U$, the spectra exhibits peaks due to prompt ejection from the surface layers in the initial track (second frame in Fig. 3). This includes initially 'ionized' species and accounts for $\sim$ $20 \%$ of the ejecta at large $d J / d x$, but dominates at very small $d J / d x$. On the other hand, the principal component of the ejecta in Fig. 5 has an energy distribution like that found in our studies of ejection from a cylindrical spike (dashed line) [10]. That is, there is a broad, quasi-thermal distribution at low ejecta energies, $E<U$, which gives way to a $\sim E^{-2}$ dependence at $E>U$ differing from thermal spike model predictions [10]. This is the ejection seen in frames 4-6 in Fig. 3. If CE contributes to sputtering, the experimental energy spectra would have a peak due to ejection of surface ions (which may be neutralized on their way to the detector). In an experiment, the size of this peak will be small and may be hidden by the background noise. The position of the peak is related to $(d J / d x)_{e f f}: K E \approx C_{1} U[l(d J / d x)]^{2}-U-E_{p o l}$, where $C_{1}$ depends strongly on the track radius, $r_{t r}$, and screening length, $a$, and slightly on the neutralization time, $\tau$. $E_{\text {pol }}$ is the energy required to extract an ion from the surface due to polarization forces. For our MD simulation, $E_{p o l}=0$, $r_{t r} \approx l_{s}$, and $C_{1} \sim 35$ for $a \approx l_{s}$. Therefore, the CE should exhibit a peak at $K E \sim 34 U$ for $[l(d J / d x)]=N_{c h}=2$. The peak in the MD simulations, in fact, appears at $K E \sim 38 U$ for $\tau=0$ in agreement with this estimate. For $\tau=\tau_{D}$ a broad peak with a height $\sim 15$ times smaller than the height of the maximum is located at $K E \sim 32 U$. 
In order to verify if the repulsive energy transferred to the lattice indeed forms a TS, energy transport at the atomic level should be extracted from these simulations [8]. When we compare the nature of the energy transport beyond $\sim 0.2 \tau_{D}$, the sputtering yield, and the ejecta energy spectra with the results from our earlier thermal spike MD simulations, the quantitative agreement is good. In both cases, the transport is not simply by thermal diffusion. At large $d J / d x$ most of the ejecta are generated by the "thermal" hot spike produced. That is, the 'ionization track' rapidly produces a heat spike which determines the subsequent energy transport, sputtering and bulk damage. This means that 'CE' and spikes are the early and late aspects of an 'ionization track' and differences in predictions occur due to the use of incorrect spike models.

\subsection{Electronic sputtering of molecular condensed gas solids}

One of the principal outstanding problems in electronic sputtering is the fact that yields for molecular condensed gas solids appear to vary roughly quadratically with the electronic stopping $(d E / d x)_{e}$, at high $(d E / d x)_{e}$, as initially

shown by Brown and co-workers (see e.g. [5] ). Since both CE and analytic TS models directly give such a dependence, they have been used to analyze the laboratory data [32]. The standard analytic TS model, which has now been shown to be incorrect [10], can be modified [35]. Here we note that our CE MD simulations confirm that a CE model can also be applied. That is, a $\mathrm{CE}$ can produce a TS at high $d J / x$. If the neutralization times and electron densities are such that $\tau \gtrsim 0.2 \tau_{D}$ and $a \sim l$ for $0.02 \tau_{D}<t<0.2 \tau_{D}$, a quadratic dependence of the yield $d J / d x$ occurs and the ejecta energy spectra are similar to those observed for molecular condensed gas solids [36,37]. Since $d J / d x \propto(d E / d x)_{e}$, the resulting yield is also quadratic in $(d E / d x)_{e}$. 
Because electron density and neutralization times are difficult to measure or evaluate theoretically, it is not clear if the above conditions are fulfilled for the tracks in molecular condensed gas solids. If the screening length varies with $(d E / d x)_{e}$ the yield might not be quadratic and the neutralization times are not well known but may be short. The sizes of the yield in the model MD simulations are consistent with the experimental yield for solid $\mathrm{O}_{2}$ at high $(d E / d x)_{e}$. However, if only Coulomb repulsion were driving sputtering, the sputtering yields of solid $\mathrm{O}_{2}$ and $\mathrm{N}_{2}$ should be close in size, since they have the same binding energy, $U$, and, one would assume, similar neutralization times and hole diffusion. However, the yield for $\mathrm{O}_{2}$ is almost one order of magnitude larger suggesting that differences in the relaxation processes are critical: differences in ionization levels, luminescence [32] and chemistry of the dissociation products [38].

\section{Summary}

We carried out MD simulations of a simplified CE model, which roughly described that when the electrons are excited by a fast ion the average track potential experienced by the ions is repulsive. Using a screened Coulomb potential with constant screening length, the simulations verified predictions that the sputtering yield is quadratic in the ionization per unit path length, $d J / d x$, created in the track of a fast, ion over a broad range of $d J / d x$.

The yield consists of two components: prompt ejecta from the track core, which dominates at small $d J / d x$, and ejection from the heat spike formed, which dominates at high $d J / d x$. That is, the potential energy was seen to

evolve into kinetic energy of the ions in the track and of the surrounding 
neutrals. This can form a cylindrical spike with a radius larger than the initial track radius producing sputtering.

The mechanism for converting electronic energy into lattice motion can be either the transfer of energy to the lattice directly by the secondary electrons or by a repulsive force. The repulsion may result when electronic recombination occurs $\left(\mathrm{O}_{2}^{+}+e \rightarrow\right.$ repulsive state $\left.\rightarrow \mathrm{O}+\mathrm{O}+\Delta E\right)$, due to the repulsive track of holes partially screened by free electrons, or by the interaction between 'excited atoms' in the track. At high excitation densities, once the energy is in the lattice motion, the evolution of this energy can be described by that spike model shown to be correct by MD [10] and hydrodynamic simulations [11], rather than the convenient but incorrect analytic diffusive spike model. In order to determine the evolution of the ion track it is crucial to accurately describe the electron screening and neutralization [8]. When neutralization is introduced, the yield can decrease. Neutralization times longer than one tenth of the Debye period are needed in order for $\mathrm{CE}$ to be effective in heating the lattice and producing sputtering. Information on electron temperature in the track, for instance from Auger electron spectra[33,39], can be used to test models. Measurements of luminescence in condensed gas solids [40], response of semiconductor detectors to radiation [41] and the survival probabilities of ions in liquids $[28,29]$ can also provide information on neutralization.

Although sputtering is a very old field of physics, the discovery of electronicallyinduced sputtering and the possibility of creating extremely high energy densities in very narrow regions will continue to test our ability to describe solid state processes under extreme conditions. 


\section{Acknowledgements}

I would like to thank B.D. Wirth for helpful comments and R.E. Johnson for his continuous collaboration and support. This work was supported by the National Science Foundation Astronomy and Chemistry Divisions, and it was partially performed under the auspices of the U. S. Department of Energy by the University of California, Lawrence Livermore National Laboratory under Contract No. W-7405-Eng-48.

\section{References}

[1] R. L. Fleischer, P. B. Price, and R. M. Walker, J. App. Phys. 36, 3645 (1965); R. L. Fleischer et al., Phys. Rev. 156, 353 (1967).

[2] C. Trautmann, S. Klaumunzer and H. Trinkaus, Phys. Rev. Lett. 85, 3648 (2000).

[3] D. Lesueur and A. Dunlop, Rad. Eff. and Def. in Solids 126, 123 and 163 (1993).

[4] R.M. Papaléo et al., Phys. Rev. B 62, 11273 (2000).

[5] R. E. Johnson and W. L. Brown, Nucl. Instrum. Meth. 198, 103 (1982).

[6] P. K. Haff, Appl. Phys. Lett. 29, 443 (1976).

[7] T. Schenkel et al, Phys. Rev. Lett. 81, 2590 (1998).

[8] E.M. Bringa and R.E. Johnson, Phys. Rev. Lett. 88, 165501 (2002).

[9] F. Seitz, Discussions Faraday Soc. 5, 271 (1949).

[10] E. M. Bringa, R. E. Johnson and M. Jakas, Phys. Rev. B 60, 15107 (1999).

[11] M. Jakas, E.M. Bringa and R.E. Johnson, Phys. Rev. B 65, 165425 (2002). 
[12] M. Toulemonde, Ch. Dufour, A. Meftah and E. Paumier, Nucl. Instr. and Meth. B 166-167, 903 (2000).

[13] L. E. Seiberling, J. E. Griffith, and T. A. Tombrello, Rad. Eff. 52, 201 (1980).

[14] R. H. Ritchie and C. Claussen, Nucl. Instr. and Meth. 198 (1982) 133.

[15] R. H. Ritchie, A. Gras-Marti, and J. C. Ashley, Proceedings of the $12^{\text {th }}$ Werner Brandt Workshop, San Sebastian, Spain, 1989.

[16] C. Watson and T. Tombrello, Rad. Eff. 89, 263 (1985).

[17] P. Stampfli and K. H. Bennemann, Appl. Phys. A 60, 191 (1996).

[18] I.S. Bitenskii, M. N. Murakhmetov and E.S. Parilis, Sov. Phys. Tech. Phys. 24, 618 (1979).

[19] L. Poth and A. W. Castleman Jr., J. Phys. Chem. A 102 (1998) 4075.

[20] S. A. Fedotov et. al, Nucl. Instr. and Meth. B 118 (1996) 724.

[21] P. Legrand, J. Morillo, and V. Pontikis, Rad. Eff. and Def. in Solids 126 (1993) 151.

[22] R. E. Walkup and Ph. Avouris, Phys. Rev. Lett. 56, 524 (1986).

[23] E. Wu, R. J. Friauf, and T. P. Armstrong, Surf. Sci. 249 (1991) 350.

[24] M. Hedström and H-P. Cheng, Phys. Rev. B 62, 2751 (2000).

[25] A. F. Lifschitz and N. R. Arista, Phys. Rev. A 57 (1998) 200.

[26] E. Nardi and Z. Zinamon, Laser Part. Beams 13 (1995) 335.

[27] S. E. Kerns, in Ionizing Radiation Effects in MOS Devices and Circuits, T. P. Ma and P. V. Dressendorfer eds., John Wiley \& Sons, New York, 1989, p. 485.

[28] W. M. Bartzac, L. D. A. Siebels, and A. Hummel, J. Phys. Chem. A 101 (1997) 8135. 
[29] A. Mozumder, J. Chem. Phys. 55 (1971) 3020, and J. Chem. Phys. 101 (1994) 10388.

[30] J. Ferrón and R. Vidal, private communication. R. Vidal, R. A. Baragiola and J. Ferrón, J. Appl. Phys. 80 (1996) 5653.

[31] M. Liu and R. E. Johnson, to be submitted.

[32] Johnson, R.E. and J. Schou, Mat. Fys. Medd. K. Dan. Vidensk. Selsk. 43, 403 (1993).

[33] Schiwietz et al., Phys. Rev. Lett. 69 (1992) 628 and Nucl. Instr. and Meth. B 146 (1998) 131.

[34] R. A. Baragiola, M. Shi, R. A. Vidal, and C. Dukes, Phys. Rev. B 58 (1998) 13212.

[35] E. M. Bringa and R. E. Johnson, Surf. Sci. 451 (2000) 108.

[36] W. L. Brown et al., Nucl. Instr. and Meth. B 1 (1984) 307.

[37] C.T. Reinmann et al., Surf. Sci. 147 (1984) 227.

[38] R. A. Baragiola, C. L. Atteberry, D. A. Bahr, and M. M. Jakas, Nucl. Instr. and Meth. B 157 (1999) 233.

[39] Z. G. Wang, C. Dufour, E. Paumier, M. Toulemonde, J. Phys. Cond. Matter 6 (1994) 6733.

[40] A. Hitachi, T. Doke and A. Mozumder, Phys. Rev. B 46 (1992) 11463.

[41] E. C. Finch, A. A. Cafolla, M. Asghar, Nucl. Instr. and Meth. 198 (1982) 547. 


\section{FIGURES}

Fig. 1. The sputtering yield from a repulsive track vs. the screening constant a. The dashed lines are from Eq. 1 .

Fig. 2. Yield vs. $\tau$, the neutralization/recombination time. The solid lines are from Eq. 1, $Y(\tau=\infty) \exp \left[-\alpha\left(\tau_{D} / \tau\right)^{p}\right]$ with $\alpha=0.75$ and $p=0.5$. A better fit can be obtained varying $\alpha$ and $p$ slowly with $\left[l_{s} d J / d x\right]$.

Figure 3. Snapshots from a MD simulation of CE. Screening constant $a \approx l_{s}$, neutralization time $\tau=2 \tau_{D}$, and $N_{c h}=2$ in the fully excited track of $r_{t r} \approx l_{s}$. $U=0.08 \mathrm{eV}$, and $\tau_{D}=0.5 \mathrm{ps}$. Charged particles have twice the size of neutrals. Color code correspond to kinetic energy of the particles (red hotter, blue colder).

Fig. 4. Depth and radial distribution of ejecta for $N_{c h}=l(d J / d x)_{e f f}=2$, $a \approx l_{s}$ and $r_{t r} \approx l_{s}$. Both $\tau=\tau_{D}$ and $\tau=20 \tau_{D}$ are shown.

Figure 5. Energy distribution of the ejecta for $\left[l_{s} d J / d x\right]=2, a \approx l_{s}$ and $r_{t r} \approx$ $l_{s}$, for $\tau=\tau_{D}$ (a) and $\tau=20 \tau_{D}$ (b). Dotted line: energy spectrum obtained adding a Maxwellian distribution to a Sigmund-Thomson distribution. High energy peaks are prompt ejecta determined by the potential energy between neighbors and $U$. 


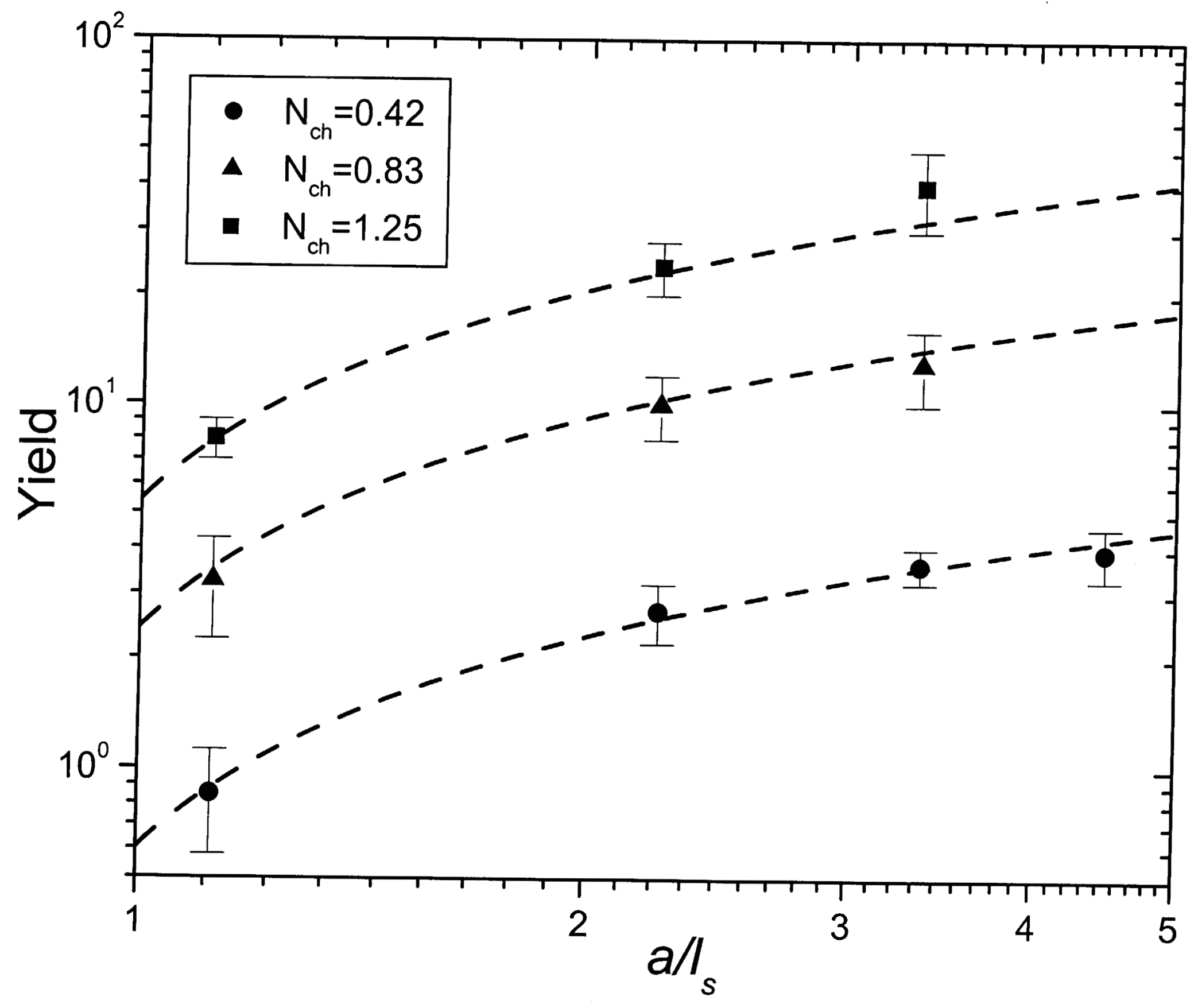

Fig 1. Bringa and Johnson 


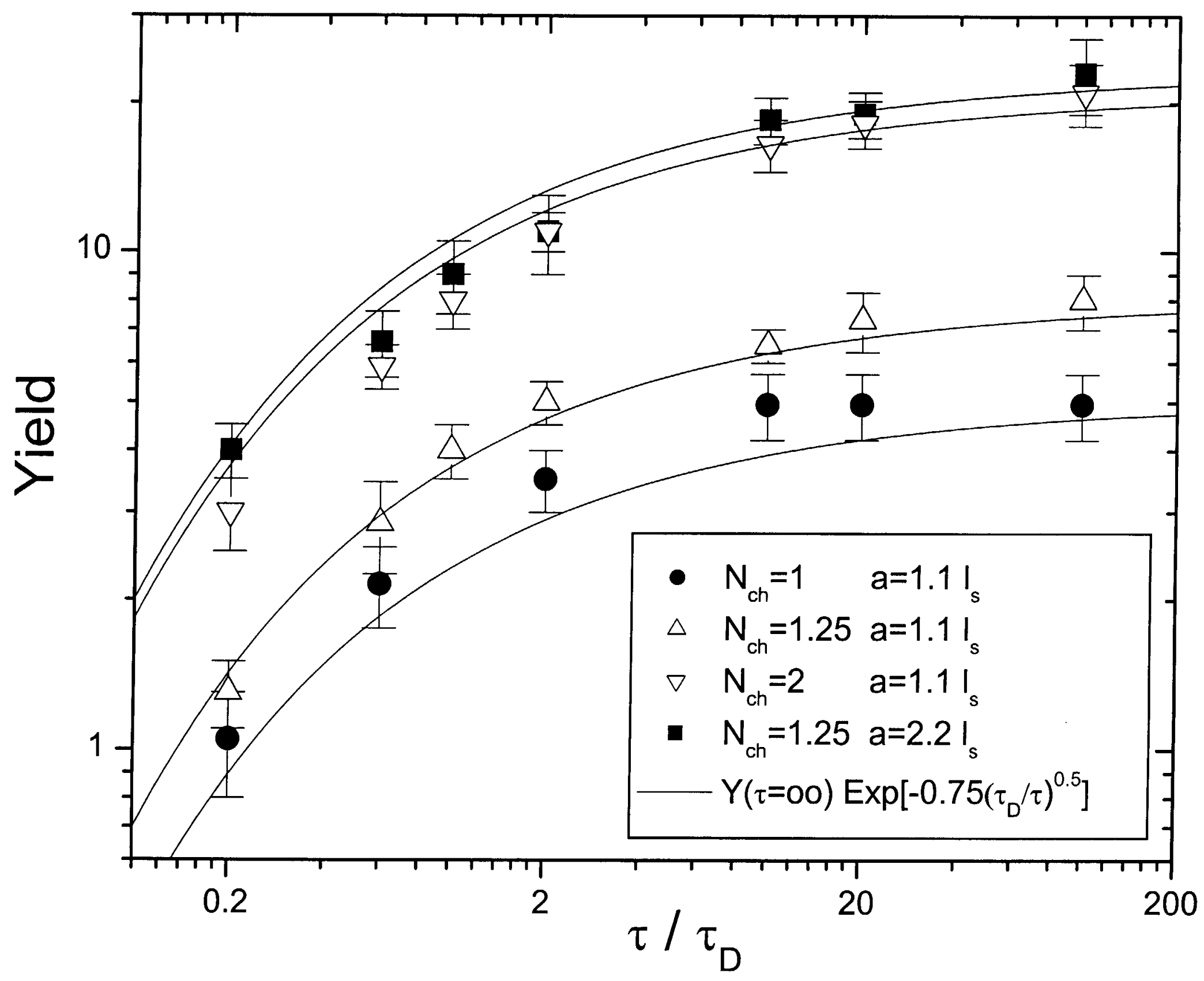

Fig 2. Bringa and Johnson 

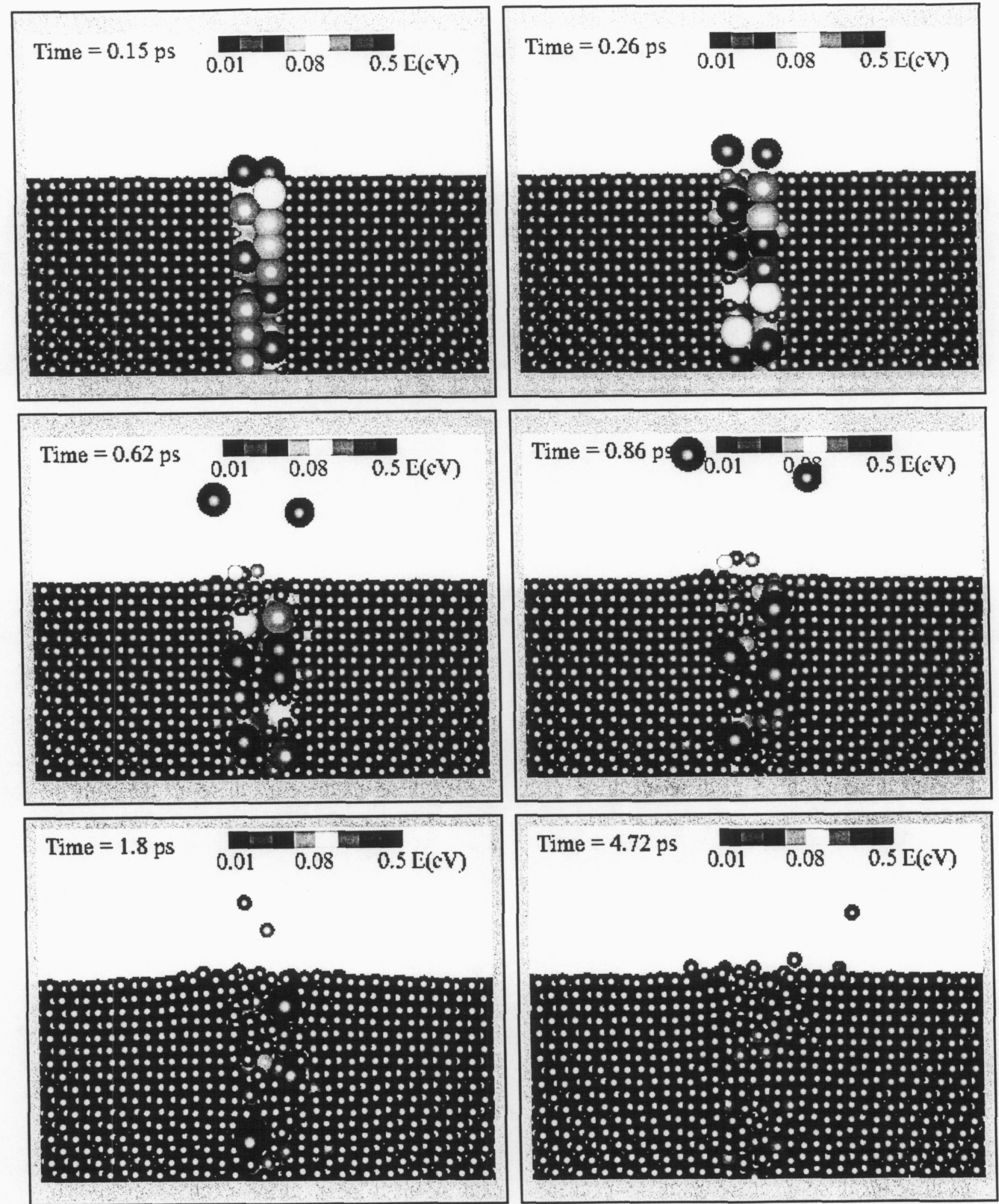

Fig. 3. Bringa and Johnson 


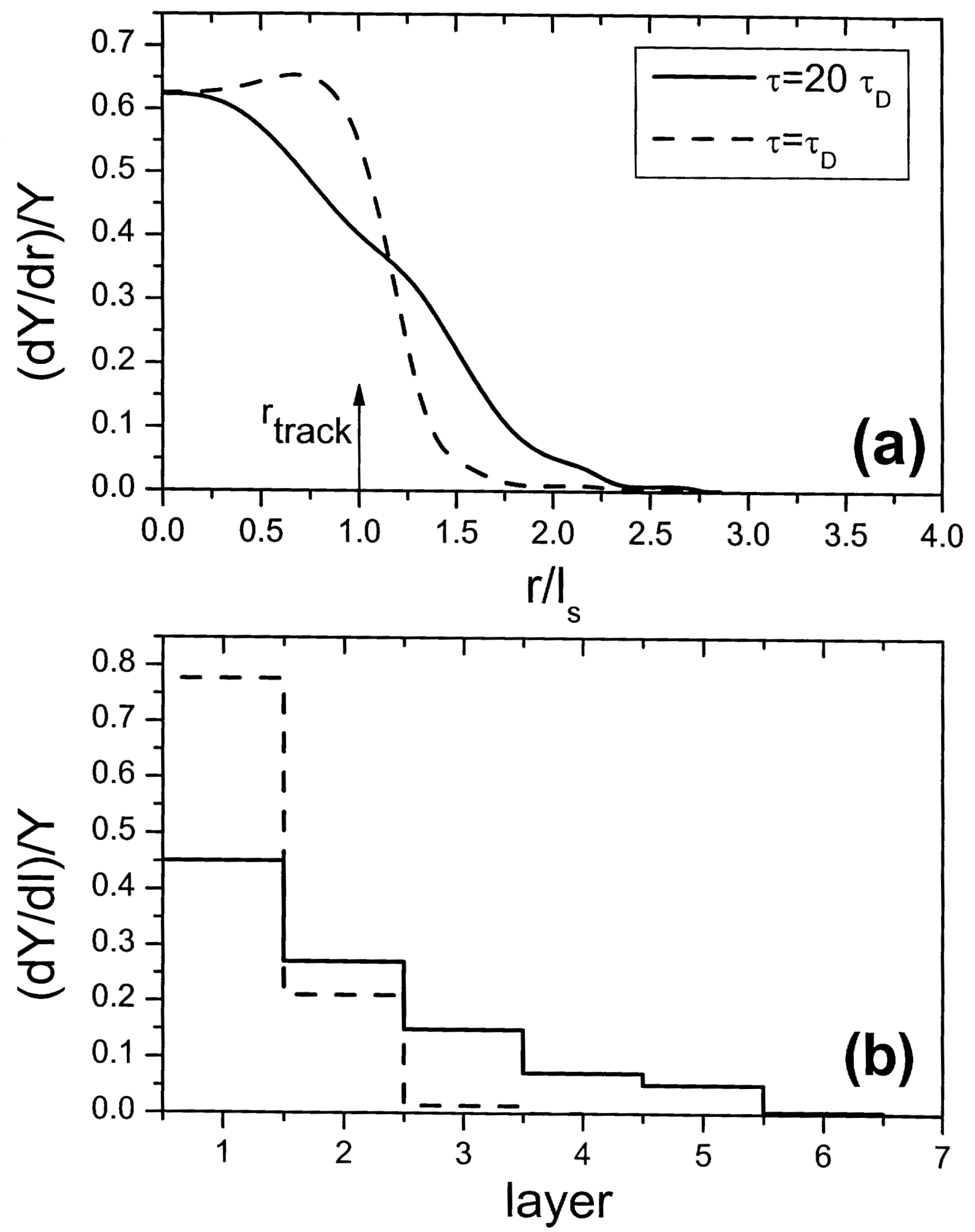

Fig. 4. Bringa and Johnson 

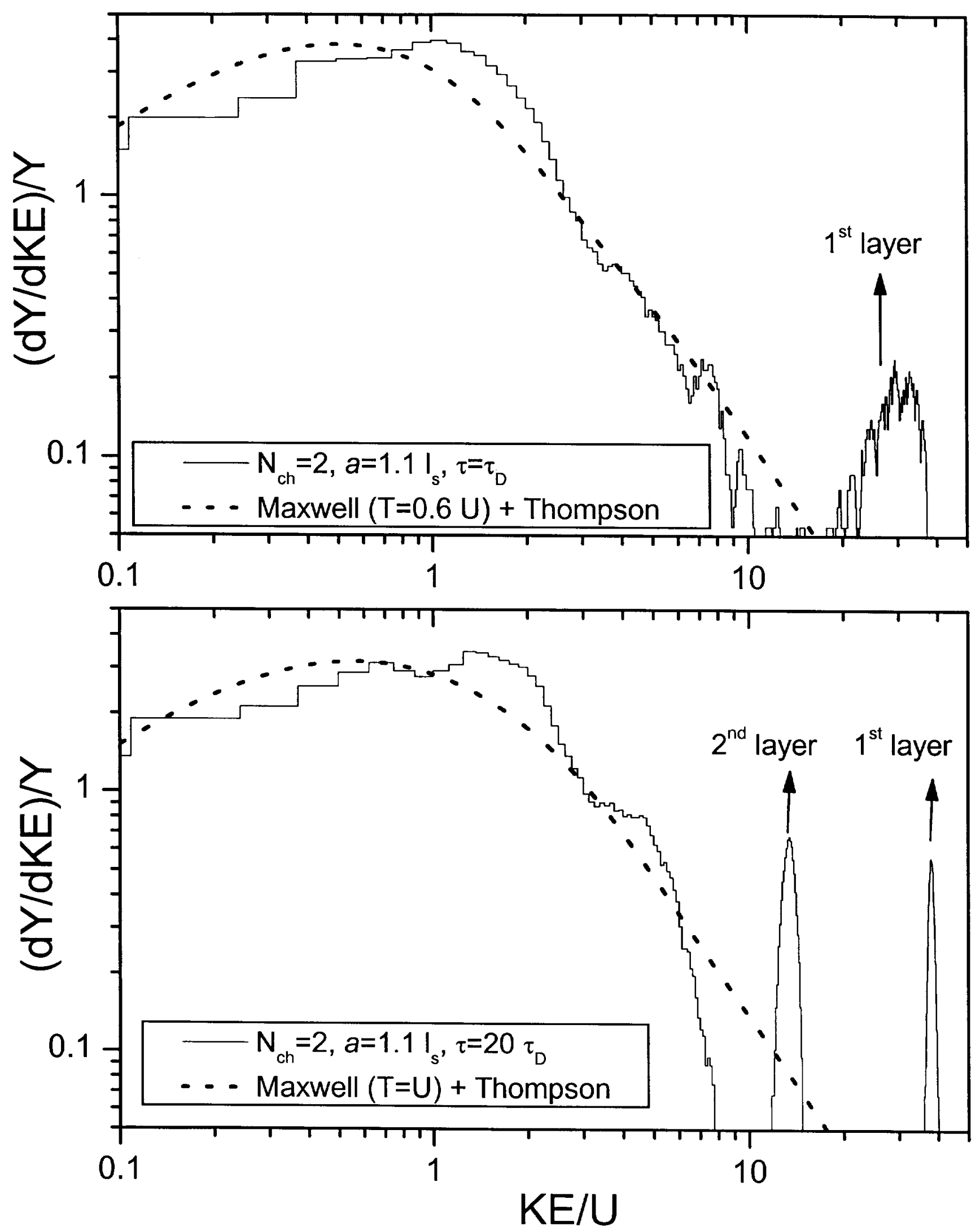

Fig. 5. Bringa and Johnson 